\title{
TILAPIA-HAUT: TECHNOLOGISCHER FORTSCHRITT BEI DER BEHANDLUNG VON VERBRENNUNGEN?
}

\section{ARTIKEL ÜBERPRÜFEN}

CABRAL, Anna Julie Medeiros ${ }^{1}$, LIMA, Camila Araújo Novais², CRUZ FILHO, Eduardo Franco Correia ${ }^{3}$, SOARES, Gabriel Lucena de Carvalho ${ }^{4}$, ESPÍNOLA, Paulo Francisco Lucena de Araújo ${ }^{5}$, ALVES, Fernanda Araújo6 ${ }^{6}$, CRUZ, Ana Suzy de Góis Melo ${ }^{7}$

CABRAL, Anna Julie Medeiros. Et al. Tilapia-Haut: technologischer Fortschritt bei der Behandlung von Verbrennungen?. Revista Científica Multidisciplinar Núcleo do Conhecimento. Jahr. 06, Hrsg. 12, Vol. 05, S. 50-64. Dezember 2021. ISSN: 2448-0959, Zugangslink:

https://www.nucleodoconhecimento.com.br/gesundheit/tilapia-haut, DOI: 10.32749/nucleodoconhecimento.com.br/gesundheit/tilapia-haut

\section{ZUSAMMENFASSUNG}

Einleitung: Verbrennung ist eine der größten Aggressionen, die der Körper erleiden kann. Der Ansatz variiert je nach Grad der Verbrennung, seit der Verwendung von Chlorhexidin, Silbersulfadiazin, Debridement von nekrotischem Gewebe, biosynthetischen Verbänden und künstlichen Häuten. Diese beiden letzteren haben jedoch hohe Kosten, so dass mehrere Studien mit dem Ziel entstanden sind, praktikablere Optionen zu suchen, wie die Verwendung von Nil-Tilapia-Haut bei

\footnotetext{
${ }^{1}$ Student des medizinischen Kurses des Universitätszentrums von João Pessoa - UNIPÊ, João Pessoa - PB. ORCID: 0000-0002-5212-5521.

${ }^{2}$ Student des medizinischen Kurses des Universitätszentrums von João Pessoa - UNIPÊ, João Pessoa - PB. ORCID: 0000-0003-4609-4260.

${ }^{3}$ Student des medizinischen Kurses des Universitätszentrums von João Pessoa - UNIPÊ, João Pessoa - PB. ORCID: 0000-0001-6684-2161.

${ }^{4}$ Student des medizinischen Kurses des Universitätszentrums von João Pessoa - UNIPÊ, João Pessoa - PB. ORCID: 0000-0002-0618-4037.

${ }^{5}$ Student des medizinischen Kurses des Universitätszentrums von João Pessoa - UNIPÊ, João Pessoa - PB. ORCID: 0000-0001-7964-2118.

${ }^{6}$ Berater. ORCID: 0000-0001-5235-5020.

${ }^{7}$ Berater. ORCID: 0000-0002-0668-0540.
} 
Verbrennungen aufgrund ihrer heilenden Eigenschaften. Allerdings hat der vorliegende Artikel als Leitfrage: Ist diese neue Methode tatsächlich ein technologischer Fortschritt, der für die Behandlung von verbrannten Patienten so wichtig ist, wie es scheint? Ziel: Die Verwendung von Nil-Tilapia-Haut bei Patienten mit Brandverletzungen zu analysieren und mit anderen im Voraus etablierten Techniken zu vergleichen. Methoden: Dies ist eine integrative bibliographische Überprüfung mit einem qualitativen Ansatz. Die Daten wurden von 2015 bis 2020 über PubMed -Datenbanken und die Virtuelle Medizinische Bibliothek (VMB) gesammelt. Ergebnisse: Die Artikel zeigen eine gute Prognose für die Anwendung von Nile Tilapia-Haut im Vergleich zu anderen aktuellen Optionen zur Behandlung von Verbrennungen mit einem signifikanten Vorteil in der Reduzierung der Anzahl der benötigten Verbände aufgrund der besseren Haftung auf der Wunde. Darüber hinaus hat es mikroskopische Eigenschaften ähnlich der menschlichen Haut, wie hohe Zugfestigkeit und Bruchdehnung, mit reduzierter Reepithelialisierung und Schmerzintensität sowie reduzierten Behandlungskosten. Fazit: Angesichts der Ergebnisse der in der vorliegenden Übersicht berichteten Literatur wird der Schluss gezogen, dass sich Studien mit Nil-Tilapia-Haut als revolutionäre Modalität mit Nutzen bei der Behandlung von Patienten mit oberflächlichen und tiefen Hautläsionen erweisen. Daher schlossen die Forscher bei der Beantwortung der Leitfrage, dass die neue Methode, ja, ein wichtiger Fortschritt auf dem Gebiet der Verbrennungsbehandlung ist, da ihre Beschäftigungsfähigkeit bestätigt wird, neben dem Nachweis eines Vorteils gegenüber einigen der wichtigsten bereits bestehenden Alternativen.

Schlüsselwörter: Tilapia, Verbrennungen, biologische Verbände.

\section{EINLEITUNG}

Die Geschwindigkeit und der Lebensstil, die von der Moderne auferlegt werden, führen dazu, dass die Häufigkeit der Verbrennungen heute zunimmt. Weit über körperliche Verletzungen hinaus sind Verbrennungen auch für wirtschaftliche Probleme verantwortlich, die mit irreparablen Schäden für Patienten und ihre 
Familien verbunden sind ( $\mathrm{HU}$ et al., 2017). Die Verbrennung ist eine der größten Aggressionen, die der menschliche Organismus erleiden kann, und es wird geschätzt, dass es in Brasilien laut Leontsinis et al. jährlich etwa 1 Million Verbrennungsunfälle gibt. (2018), ob es sich um einen einfachen Hitzschlag, Verbrennungen ersten Grades, bis zur totalen Zerstörung der Haut (Epidermis und Dermis) und angrenzender Gewebe handelt, wie bei Verbrennungen dritten Grades (ALVES et al., 2015).

Wenn wir die Behandlung von Verbrennungen in Brasilien betrachten, stehen wir vor zwei Realitäten: der des öffentlichen und des privaten Netzwerks. In den Behandlungszentren des öffentlichen Netzwerks ist das Verhalten in Bezug auf Verbände in Läsionen zweiten Grades das tägliche Bad mit 2\% Chlorhexidin und der Verband wird mit dem topischen antimikrobiellen Silbersulfadiazin bei $1 \%$ bis zur vollständigen Reparatur der Läsion durchgeführt; während bei Verbrennungen dritten Grades nekrotisches Gewebe in mehreren Stufen debridiert wird, und dann wird der Verband mit $1 \%$ Silbersulfadiazin durchgeführt, bis das Wundbett für die Transplantation vorbereitet ist. Auf der anderen Seite können im privaten Netzwerk unter Berücksichtigung der unterschiedlichen finanziellen Bedingungen jedes Patienten biosynthetische Verbände und künstliche Haut, importierte und teure Optionen verwendet werden (ALVES et al., 2015).

Bei der Analyse der in Europa und den Vereinigten Staaten erlebten Realität beinhaltet die Behandlung jedoch häufig die Verwendung homologer Haut (unter Verwendung von Hautbänken), Herherologen (tierischen Ursprungs), biosynthetischer Verbände und künstlicher Dermis (ALVES et al., 2015). In Brasilien gibt es immer noch einige Konfrontationen, um die gleiche Praktikabilität dieser anderen Länder zu erreichen. Kulturell gibt es immer noch große Widerstände gegen Haut spenden, was die Verfügbarkeit des Stoffes einschränkt, um die große Nachfrage zu befriedigen. Darüber hinaus wäre es unter Bezugnahme auf das Gesundheitsministerium notwendig, 13 Hautbanken im gesamten Staatsgebiet zu verteilen, es sei jedoch nur fünf (São Paulo, Curitiba, Rio de Janeiro, Porto Alegre und Recife - das behindert ist). Zusätzlich zu diesen Einschränkungen hat Brasilien 
noch nie eine Tierhaut bei der Nationale Gesundheits Überwachungsbehörde (ANVISA) registriert und von Gesundheit Unic-System (SUS) zur Verfügung gestellt, was auch die Verwendung von heterologer Haut bei verbrannten Patienten einschränkt (LEONTSINIS et al., 2018).

So sind mehrere Studien mit dem Ziel entstanden, nach Verbänden zu suchen, die bei verbrannten Patienten verwendet werden können. Diese Verbände zielen darauf $\mathrm{ab}$, die Auswirkungen der Kontamination auf Läsionen zu reduzieren, den Heilungsprozess zu begünstigen und bessere ästhetische Ergebnisse zu erzielen. Im Idealfall gute Flexibilität und Haftung am Bett, Widerstandsfähigkeit gegen Dehnung, einfache Handhabung, Fähigkeit, Schmerzen zu unterdrücken, niedrige Kosten und leicht zu erhalten, sowie hydroelektrolytische Verluste, bakterielle Kontamination, begünstigt die Epithelisierung von Verbrennungen und sorgt für die Bildung von geeignetem Granulationsgewebe.Eine der Optionen ist die Suche nach temporären Hautersatzstoffen und Verbänden aus synthetischen oder biosynthetischen Materialien, da sie die Häufigkeit des Verbandwechsels reduzieren, jedoch die hohen Kosten und ihre Unwirksamkeit für tiefe Verbrennungen die Suche nach biologischen Materialien als praktikable Alternativen stimulierten (COSTA et al., 2019; MARCELO und BRANDT, 2019).

In diesem Zusammenhang, im Jahr 2011 der plastische Chirurg aus Pernambuco Marcelo Borges, nachdem er im Jornal do Commercio de Pernambuco einen Artikel über die Verwendung der Haut der Nil-Tilapia-Art (Oreochromis niloticus) im Handwerk gesehen hatte, einem der am meisten kultivierten Fische kam in Brasilien erstmals auf die Idee, diese Haut zur Behandlung von Verbrennungen einzusetzen. Aber erst 2014 teilte er die Idee mit dem plastischen Chirurgen von Ceará Edmar Maciel und begann dann studien über die Verwendung von Nil-Tilapia-Haut bei Verbrennungen. Der Forscher Odorico Moraes, Präsident des Zentrums für Forschung und Entwicklung von Arzneimitteln (NPDM) an der Bundesuniversität Ceará (UFC), und der plastische Chirurg von Goiás Nelson Piccolo (LIMA JUNIOR et al.) wurden ebenfalls integriert, um die Koordination der Arbeit zu übernehmen. 2017). 
Wie von Lima Junior (2017) erzählt, zeigt die Haut von Nil-Tilapia eine Epidermis, die mit einem geschichteten Pflasterepithel bedeckt ist, gefolgt von ausgedehnten Kollagenschichten. Dank seiner Eigenschaft, die meisten Gewebe zu führen und zu definieren, ist es als ein großartiger Bestandteil von Biomaterialien konfiguriert, neben der Ermöglichung der biologischen Abbaubarkeit und Biokompatibilität, die seine Anwendung begünstigen (LIMA JUNIOR, 2017; LIMA JUNIOR et al., 2019b, 2020b).

Die richtige Frage ist jedoch: "Ist diese neue Methode tatsächlich ein technologischer Fortschritt, der für die Behandlung von verbrannten Patienten so wichtig ist, wie es scheint?" Ziel ist es dann, durch diese Studie zu analysieren, ob Nil-Tilapia-Haut eine praktikable Alternative für die Anwendung bei Patienten mit Brandverletzungen ist, sowie mit anderen bereits verwendeten Techniken zu vergleichen, um die Vorteile der Einführung dieser neuen Methode zu überprüfen.

Genauer gesagt zielt diese Studie darauf ab, die guten Vorräte von TilapiaHautverbänden bei der Behandlung von Verbrennungen nachzuweisen, die physikalisch-chemischen Eigenschaften von Tilapia mit den Vorteilen des Verbandes zu korrelieren und die Nichtunterlegenheit von Tilapia-Hautverbänden im Vergleich zu anderen Lösungen auf dem Markt aufzudecken. Neben der Anstiftung zur Durchführung weiterer wissenschaftlicher Forschung, um robustere Ergebnisse zu diesem Thema zu erhalten.

\section{METHODIK}

Es bezieht sich auf eine integrative Literaturrecherche, mit einem qualitativen Ansatz, mit deskriptiven Zweck nationaler und internationaler Studien. Darüber hinaus hat es einen angewandten Charakter, mit der Verwendung eines bibliographischen Verfahrens für die Analyse der jeweiligen Daten, die im Zusammenhang mit der Verwendung von Tilapia-Haut bei verbrannten Patienten gesammelt wurden.

Die vorliegende Studie wurde durch die Suche nach Artikeln durch den Zugriff auf pubmed-Datenbanken und die Virtuelle Gesundheitsbibliothek (VHL) durchgeführt. 
Zunächst wurden die für das ausgewählte Thema relevanten Deskriptoren gesammelt, die Vokabular der Deskriptoren in den Gesundheitswissenschaften (DeCS) sind. Die gewählten Begriffe waren "Tilapia", "brennt" und "Biologische Verbände" in den Sprachen: Portugiesisch, Englisch und Spanisch. Diese Deskriptoren wurden mit den booleschen Operatoren "OR" und "AND" in Verbindung gebracht, indem die folgende Forschungsformen ausgearbeitet wurde: "(Tilápia OR Tilapia OR Tilapia) AND (Queimaduras OR Burns OR Quemaduras) AND (Curativos Biológicos OR Biological Dressings OR Apósitos Biológicos)".

So wurden unter Anwendung der oben genannten Formel insgesamt 31 Artikel in den ausgewählten Datenbanken gefunden, 14 pubmed-Artikel und 17 im VHL verfügbar. Die Einschlusskriterien waren: vollständig verfügbare Artikel, die das Thema im Zusammenhang mit der integrativen Überprüfung darstellen, und Artikel, die in den in den Jahren 2015, 2016, 2017, 2018, 2019, 2020 und 2021 veröffentlichten und indizierten Datenbanken veröffentlicht und indiziert wurden und die Sprache in Englisch, Portugiesisch und Spanisch präsentierten. Darüber hinaus wurden die Artikel, die dupliziert wurden, keinen vollständigen Inhalt hatten und die Forschungsziele nicht erfüllten, von der integrativen Überprüfung ausgeschlossen.

Die integrative bibliographische Überprüfung ist nicht praktikabel und erfordert daher keine Einreichung bei der Forschungsethikkommission (CEP). Aus dieser Perspektive wurde eine Tabelle erstellt, die die wichtigsten Informationen der verwendeten Bibliographien enthält und die Daten enthält, die sich auf den Titel, die Autoren, das Erscheinungsjahr, das Land und die wichtigsten Ergebnisse der einzelnen Bibliographien beziehen. 


\section{ERGEBNISSE}

Tabelle 1: Verwendung von Tilapia-haut bei Verbrennungen: theoretische Grundlagen und praktische Implikationen.

\begin{tabular}{|c|c|c|c|}
\hline & Titel & $\begin{array}{l}\text { Autoren, } \\
\text { Erscheinungsjah } \\
\text { r und Land }\end{array}$ & Wichtigste Ergebnisse \\
\hline $\begin{array}{l}\text { ARTIKE } \\
\text { L } 1\end{array}$ & $\begin{array}{l}\text { Uso da pele de tilápia } \\
\text { (Oreochromis } \\
\text { niloticus), como } \\
\text { curativo biológico } \\
\text { oclusivo, no tratamento } \\
\text { de queimaduras }\end{array}$ & $\begin{array}{l}\text { Lima Júnior et al., } \\
\text { 2017, Brasil }\end{array}$ & $\begin{array}{l}\text { Die Studie zeigte, dass } \\
\text { Tilapia-Haut bei } \\
\text { Labortest Ratten eine } \\
\text { beträchtliche Haftung an } \\
\text { Brandwunden aufweist, } \\
\text { mit positiven } \\
\text { Ergebnissen während } \\
\text { der Heilung, ohne } \\
\text { signifikante Mutationen } \\
\text { im biochemischen und } \\
\text { hämatologischen } \\
\text { Bereich der Leber- und } \\
\text { Nierenfunktion, was } \\
\text { eine Möglichkeit des } \\
\text { biologischen Verbandes } \\
\text { darstellt. }\end{array}$ \\
\hline $\begin{array}{l}\text { ARTIKE } \\
\text { L } 2\end{array}$ & $\begin{array}{l}\text { Xenoenxerto (pele da } \\
\text { Tilápia-do-Nilo) e } \\
\text { hidrofibra com prata no } \\
\text { tratamento das } \\
\text { queimaduras de II grau } \\
\text { em adultos }\end{array}$ & $\begin{array}{l}\text { De Miranda e } \\
\text { Brandt, 2019, } \\
\text { Brasil }\end{array}$ & $\begin{array}{l}\text { Die Forschung zeigte, } \\
\text { dass Nil-Tilapia in der } \\
\text { Funktion des okklusiven } \\
\text { biologischen Verbandes } \\
\text { bei der Behandlung von } \\
\text { Verbrennungen zweiten } \\
\text { Grades bei } \\
\text { Erwachsenen wirksam }\end{array}$ \\
\hline
\end{tabular}




\begin{tabular}{|c|c|c|c|}
\hline & & & $\begin{array}{l}\text { ist, mit ähnlichen Mitteln } \\
\text { in Bezug auf } \\
\text { Behandlungszeit, } \\
\text { Schmerz Referenz und } \\
\text { Ersatzbedarf, in Bezug } \\
\text { auf die Behandlung von } \\
\text { Patienten mit Aquacel } \\
\text { AG®. }\end{array}$ \\
\hline $\begin{array}{l}\text { ARTIKE } \\
\text { L } 3\end{array}$ & $\begin{array}{l}\text { Avaliação } \\
\text { microscópica, estudo } \\
\text { histoquímico e análise } \\
\text { de propriedades } \\
\text { tensiométricas da pele } \\
\text { de tilápia do Nilo }\end{array}$ & $\begin{array}{l}\text { Alves et al., 2015, } \\
\text { Brasil }\end{array}$ & $\begin{array}{l}\text { Tilapia-Haut weist } \\
\text { Bündel von dichten } \\
\text { Kollagenfasern auf, } \\
\text { vorwiegend Typ 1, die } \\
\text { mikroskopische } \\
\text { Eigenschaften } \\
\text { aufweisen, die denen } \\
\text { der menschlichen Haut } \\
\text { ähneln, sowie eine hohe } \\
\text { Zugfestigkeit und } \\
\text { Bruchdehnung, was sie } \\
\text { als Biomaterial in der } \\
\text { regenerativen Medizin } \\
\text { vielversprechend macht. }\end{array}$ \\
\hline $\begin{array}{l}\text { ARTIKE } \\
\text { L } 4\end{array}$ & $\begin{array}{l}\text { Innovative Burn } \\
\text { Treatment Using } \\
\text { Tilapia Skin as a } \\
\text { Xenograft: A Phase II } \\
\text { Randomized Controlled } \\
\text { trial }\end{array}$ & $\begin{array}{l}\text { Lima Júnior et al., } \\
\text { 2020, Brasil }\end{array}$ & $\begin{array}{lr}\text { Die randomisierte } \\
\text { klinische } & \text { Studie } \\
\text { verwendete } & \text { eine } \\
\text { Stichprobe von } & 62 \\
\text { Teilnehmern und zeigte, } \\
\text { dass es eine } \\
\text { Verringerung gab: } \\
\text { Zeit die } \\
\text { Reepithelisierung, die }\end{array}$ \\
\hline
\end{tabular}




\begin{tabular}{|c|c|c|c|}
\hline & & & $\begin{array}{l}\text { Schmerzintensität, die } \\
\text { Menge an Ästhetik / } \\
\text { Analgetika und die } \\
\text { Notwendigkeit, die } \\
\text { Verbände im Vergleich } \\
\text { zur Kontrollgruppe zu } \\
\text { wechseln, daher zeigte } \\
\text { Tilapia-Haut Vorteile. }\end{array}$ \\
\hline $\begin{array}{l}\text { ARTIKE } \\
\text { L } 5\end{array}$ & $\begin{array}{l}\text { Tratamento de } \\
\text { queimaduras de } \\
\text { segundo grau profundo } \\
\text { em abdômen, coxas e } \\
\text { genitália: uso da pele } \\
\text { de tilápia como um } \\
\text { xenoenxerto }\end{array}$ & $\begin{array}{l}\text { Lima Júnior et al., } \\
\text { 2020, Brasil }\end{array}$ & $\begin{array}{lr}\begin{array}{l}\text { Der Fallbericht } \\
\text { dazu bei, }\end{array} & \text { trug } \\
\text { Einschränkungen } & \text { im } \\
\text { Zusammenhang mit } & \text { Zugang } \\
\text { dem zu } \\
\text { unzugänglicheren } \\
\text { anatomischen } \\
\text { Bereichen für die } \\
\text { Verwendung } \\
\text { verringern, zu } \\
\text { präsentierte } \\
\text { Ergebnisse bei der } \\
\text { Anwendung in den } \\
\text { Genitalien und im } \\
\text { Leistenbereich. Es ist } \\
\text { eine Option für hohe } \\
\text { Verfügbarkeit } \\
\text { einfache Anwendung. }\end{array}$ \\
\hline $\begin{array}{l}\text { ARTIKE } \\
\text { L } 6\end{array}$ & $\begin{array}{l}\text { Marine Collagen } \\
\text { Peptides from the Skin } \\
\text { of Nile Tilapia } \\
\text { (Oreochromis } \\
\text { niloticus): }\end{array}$ & $\begin{array}{l}\text { Zhang Hu et al., } \\
\text { 2017, China }\end{array}$ & $\begin{array}{l}\text { Der in vitro und in vivo } \\
\text { Scraping-Assay zeigte } \\
\text { signifikante } \\
\text { Konsequenzen beim } \\
\text { Abschluss des Kratzens }\end{array}$ \\
\hline
\end{tabular}




\begin{tabular}{|c|c|c|c|}
\hline & $\begin{array}{l}\text { Characterization and } \\
\text { Wound Healing } \\
\text { Evaluation }\end{array}$ & & $\begin{array}{l}\text { mit Tilapia-Haut, wobei } \\
\text { sich die Evolution im } \\
\text { Heilungsprozess durch } \\
\text { tiefe partielle } \\
\text { Dickenverbrühung bei } \\
\text { den getesteten } \\
\text { Kaninchen als } \\
\text { vielversprechende } \\
\text { Behandlung darstellen. }\end{array}$ \\
\hline $\begin{array}{l}\text { ARTIKE } \\
\text { L } 7\end{array}$ & $\begin{array}{l}\text { A Randomized } \\
\text { Comparison Study of } \\
\text { Lyophilized Nile Tilapia } \\
\text { Skin and Silver- } \\
\text { Impregnated Sodium } \\
\text { Carboxymethylcellulos } \\
\text { e for the Treatment of } \\
\text { Superficial Partial- } \\
\text { Thickness Burns }\end{array}$ & $\begin{array}{l}\text { Lima Júnior, et al., } \\
\text { 2020, Brasil }\end{array}$ & $\begin{array}{l}\text { Diese Pilotstudie zeigte, } \\
\text { dass es keine } \\
\text { Unterlegenheit } \\
\text { gegenüber } \\
\text { lyophilisierter Tilapia- } \\
\text { Haut als therapeutische } \\
\text { Option bei } \\
\text { Verbrennungen im } \\
\text { Vergleich zu dem } \\
\text { silberimprägnation } \\
\text { schmutzigen } \\
\text { Carboxymethylcellulose } \\
\text {-Verband } \\
\text { Wirksamkeit } \\
\text { Sicherheit gibt, was die } \\
\text { Entwicklung } \\
\text { komplexerer } \\
\text { randomisierter klinischer } \\
\text { Studien in der Zukunft } \\
\text { ermöglicht. }\end{array}$ \\
\hline $\begin{array}{l}\text { ARTIKE } \\
\text { L } 8\end{array}$ & $\begin{array}{l}\text { Elaboração de } \\
\text { protocolo }\end{array}$ & $\begin{array}{l}\text { Leontsinis et al., } \\
\text { 2018, Brasil }\end{array}$ & $\begin{array}{ll}\text { Der Artikel zeigte die } \\
\text { Bedeutung } & \text { der }\end{array}$ \\
\hline
\end{tabular}




\begin{tabular}{|c|c|c|c|}
\hline & $\begin{array}{l}\text { implementação e } \\
\text { funcionamento do } \\
\text { primeiro banco de pele } \\
\text { animal do Brasil: } \\
\text { Relato de experiência }\end{array}$ & & $\begin{array}{l}\text { Entwicklung und } \\
\text { Umsetzung von } \\
\text { Protokollen für die } \\
\text { weltweit erste } \\
\text { Wassertierhautbank auf } \\
\text { und verstärkte die } \\
\text { Notwendigkeit, die } \\
\text { Standardisierung des } \\
\text { Systems zu etablieren. }\end{array}$ \\
\hline $\begin{array}{l}\text { ARTIKE } \\
\text { L } 9\end{array}$ & $\begin{array}{l}\text { Use of Tilapia Skin as a } \\
\text { Xenograft for Pediatric } \\
\text { Burn Treatment: A } \\
\text { Case Report }\end{array}$ & $\begin{array}{l}\text { Costa et al., 2019, } \\
\text { Estados Unidos }\end{array}$ & $\begin{array}{l}\text { Das Tilapia-Haut- } \\
\text { Xenotransplantat im } \\
\text { Fallbericht eines 3- } \\
\text { jährigen Brandopfers } \\
\text { zeigte positive } \\
\text { Ergebnisse } \\
\text { erheblichen mit } \\
\text { und sozialen } \\
\text { Auswirkungen auf das } \\
\text { Gesundheitssystem. }\end{array}$ \\
\hline $\begin{array}{l}\text { ARTIKE } \\
\text { L } 10\end{array}$ & $\begin{array}{l}\text { Innovative treatment } \\
\text { using tilapia skin as a } \\
\text { xenograft for partial } \\
\text { thickness burns after a } \\
\text { gunpowder explosion }\end{array}$ & $\begin{array}{l}\text { Lima Júnior et al., } \\
\text { 2019, Inglaterra }\end{array}$ & $\begin{array}{l}\text { Der Fallbericht zeigt } \\
\text { einen 23-jährigen } \\
\text { männlichen Patienten } \\
\text { mit oberflächlichen und } \\
\text { tiefen Verbrennungen } \\
\text { an der rechten bzw. } \\
\text { linken oberen } \\
\text { Extremität. Mit der } \\
\text { Verwendung von } \\
\text { Tilapia-Haut wurde die } \\
\text { Reepithelialisierung in } \\
\text { kurzer }\end{array}$ \\
\hline
\end{tabular}




\begin{tabular}{|c|c|c|c|}
\hline & & & $\begin{array}{l}\text { durchgeführt, ohne } \\
\text { Nebenwirkungen und } \\
\text { notwendigkeit eines } \\
\text { Verbandwechsels. }\end{array}$ \\
\hline $\begin{array}{l}\text { ARTIKE } \\
\text { L } 11\end{array}$ & $\begin{array}{lr}\text { Pediatric } & \text { Burn } \\
\text { Treatment } & \text { Using } \\
\text { Tilapia Skin as a } & \\
\text { Xenograft } & \text { for } \\
\text { Superficial } & \text { Partial- } \\
\text { Thickness Wounds: A } & \\
\text { Pilot Study }\end{array}$ & $\begin{array}{l}\text { Lima Júnior, } 2020 \text {, } \\
\text { Inglaterra }\end{array}$ & $\begin{array}{l}\text { Die Verwendung von } \\
\text { Tilapia-Haut wurde als } \\
\text { zusätzliche Ressource } \\
\text { zusammen mit } \\
\text { Silbersulfadiazin } \\
\text { verwendet. Die Studie } \\
\text { ergab, dass sie nicht } \\
\text { nur den Patienten } \\
\text { Vorteile brachte, } \\
\text { sondern auch die } \\
\text { Behandlungskosten } \\
\text { sowie die } \\
\text { Gesundheitsfachkräfte } \\
\text { senkte, wie z. B. die } \\
\text { Arbeitsbelastung. }\end{array}$ \\
\hline $\begin{array}{l}\text { ARTIKE } \\
\text { L } 12\end{array}$ & $\begin{array}{l}\text { Comprehensive } \\
\text { Assessment of Nile } \\
\text { Tilapia Skin } \\
\text { (Oreochromis niloticus) } \\
\text { Collagen Hydrogels for } \\
\text { Wound Dressings }\end{array}$ & $\begin{array}{l}\text { Ge et al., 2020, } \\
\text { China }\end{array}$ & $\begin{array}{l}\text { Der Kollagen-Hydrogel- } \\
\text { Verband zeigte die } \\
\text { Förderung der Bildung } \\
\text { von Epidermisschichten } \\
\text { und der Reifung der } \\
\text { Hautanhänge und } \\
\text { erwies sich als } \\
\text { ausgezeichneter neuer } \\
\text { Verband der } \\
\text { Wirksamkeit bei diefen } \\
\text { Behandlung von tit } \\
\text { Verbrennungen. }\end{array}$ \\
\hline
\end{tabular}




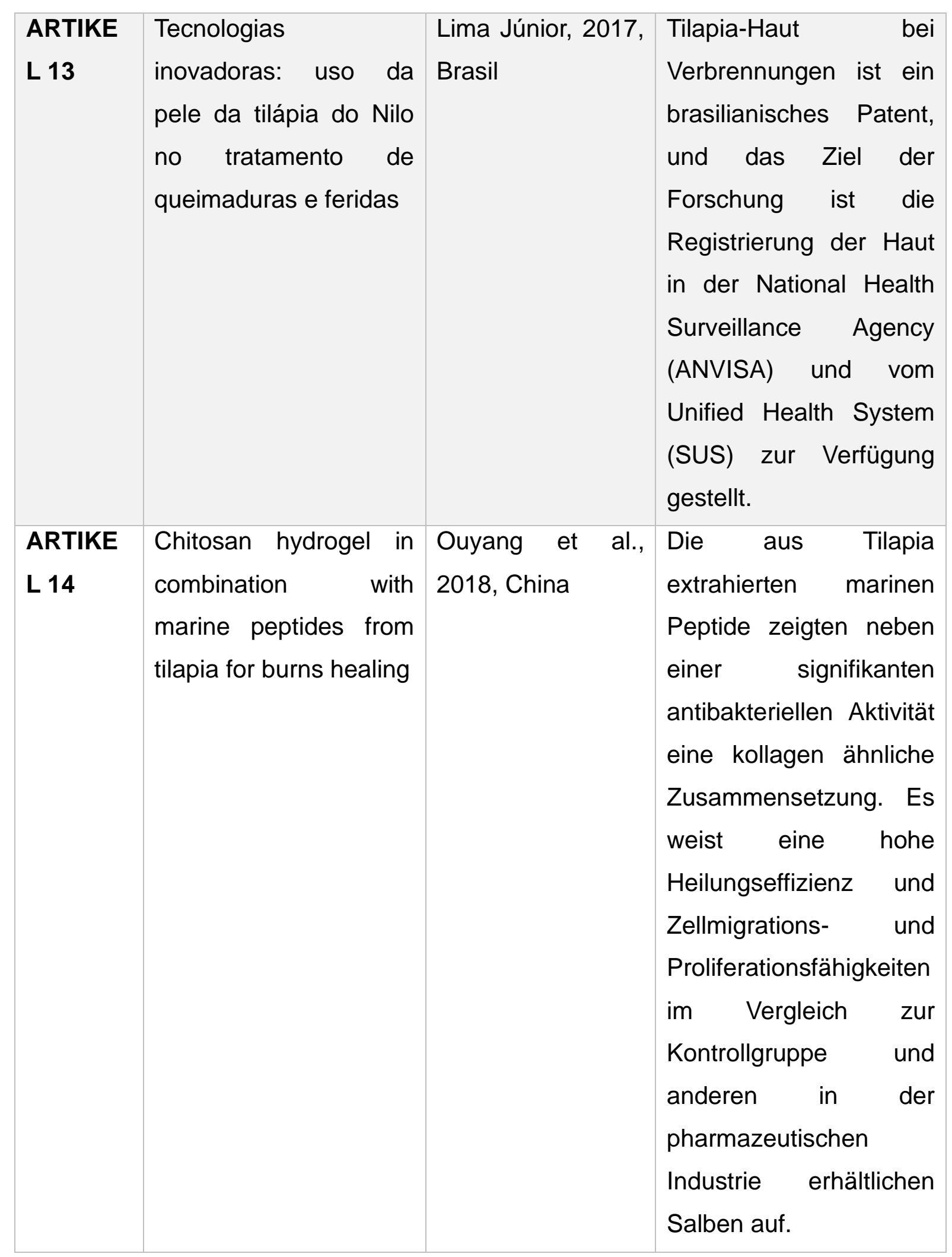

Quelle: Entwickelt vom Autor (2021) 
Die Überprüfung umfasste 14 Artikel, von denen zwei (14,3\%) über die Ergebnisse mit Tierversuchen, 14,3\% über eine vergleichende Analyse zwischen traditionellen Behandlungen und der Verwendung von Tilapia-Haut und 21,4\% über die Mechanismen der Zusammensetzung und Eigenschaften in Bezug auf die menschliche Haut berichteten. Die Verwendung von randomisierten Studien machte 7,1\% der Artikel aus, 28,6\% präsentierten die Ergebnisse in Fallberichten und 14,3\% erklärten die Entwicklung der Technik bis zu ihrer heutigen Bedeutung in den Hautbanken.

Die Studie, die im Zentrum für Verbrennungsbehandlung des Dr. José Frota Instituts, Öffentliches Krankenhaus in Fortaleza, Ceará, Brasilien, durchgeführt wurde, umfasste 62 Patienten, die zwischen Oktober 2016 und September 2017 ins Krankenhaus eingeliefert wurden und Opfer von Verbrennungen waren. Bewertet wurden: die Anzahl der Okklusivverbandwechsel und die Anzahl der Tage bis zur Wundheilung, Einsatz von Analgetika oder Anästhetika, Schmerzempfindlichkeit, Verbesserung der Verbrennung beim Abnehmen des Verbandes. Die Studie wertete die Daten aus und teilte die Teilnehmer ein nach: Geschlecht, Alter, Unfallverursacher, Körperoberfläche und Verbrennungs Segmente, und dafür, unterteilt in drei Klassifikationen, A - ambulante Patienten mit oberflächlichen Teildickenverbrennungen unter $10 \%$, B - stationäre Patienten mit derselben von 10 bis $20 \%$, C - stationäre Patienten mit tiefen Verbrennungen teilweiser Dicke von 5 bis $15 \%$, und jede dieser Klassifikationen wurde nach dem Zufallsprinzip in zwei Behandlungsgruppen eingeteilt: 48,4\% $(n=30)$ Testgruppe unter Verwendung von Tilapia-Haut als Xenotransplantat und 51,6\% ( $n=32)$ Kontrollgruppe: konventionell mit Sulfadiazin-Creme. Der Haupt Verbrennungs mechanismus in der Testgruppe (Klassifikationen A, B und C mit Tilapia-Haut als Behandlung) war durch heiße Flüssigkeiten 56,25\% $(n=18)$. Die Bewertung der Schmerzintensität war in der Testgruppe geringer als in der Kontrollgruppe. Die Anzahl der während der Behandlung benötigten Verbände war in der Testgruppe geringer und betrug 2,08 \pm 0,28 (A), 2,33 $\pm 0,71$ (B), 6,10 $\pm 2,02$ (C), während in der Kontrollgruppe 5,80 $\pm 0,42$ (A), 11,00 \pm 0,47 (B), 20,20 $\pm 1,69$ (C) unter Berücksichtigung des Wertes von $P$ als 0,0001. In Bezug auf die Anzahl der Tage bis zur vollständigen Reepithelisierung 
zeigte die Testgruppe die besten Ergebnisse mit: 9,77 $\pm 0,83$ (A), 10,56 $\pm 1,13$ (B), 18,10 \pm 0,99 (C), während die Kontrollgruppe: 11,20 $\pm 0,63(A), 11,70 \pm 0,67$ (B), $21,30 \pm 1,42 @$, unter Berücksichtigung von ungefähr $P<0,0147$ (LIMA JUNIOR et al., 2020a)

Eine weitere Studie, die im Zentrum für Verbrennungsbehandlung in Fortaleza, Ceará, Brasilien, durchgeführt wurde, wählte 30 Kinder im Alter von 2 bis 12 Jahren aus, die zwischen Mai 2017 und März 2018 ins Krankenhaus eingeliefert wurden. Die verwendeten Variablen waren: die Gesamtzahl der Tage bis zur vollständigen Heilung der Verbrennung und die Anzahl der verwendeten Verbände. Die Patienten wurden in zwei Gruppen eingeteilt, wobei 50 \% der Tilapia-Hauttest Gruppe und der Rest der Silbersulfadiazin Gruppe zugeordnet wurden. Die durchschnittliche Anzahl der Tage bis zur vollständigen Reepithelisierung betrug 10,47 $\pm 0,74$ in der Silbersulfadiazin-Gruppe und 10,07 $\pm 0,46$ in der Tilapia-Haut-Gruppe. Der Unterschied zwischen den beiden Gruppen war statistisch nicht signifikant $(P=$ 0,0868). Die Anzahl der Verbände unter Narkose, die bei Patienten, die mit TilapiaHaut behandelt wurden, durchgeführt wurde, war geringer als bei Freiwilligen, die mit Silbersulfadiazin behandelt wurden. Auch bei der Anzahl der Verbände ohne Anästhesie zeigte sich ein statistisch signifikanter Unterschied. Schließlich war die Gesamtzahl der Verbände in der Tilapia-Hauptgruppe $(3,00 \pm 0,76)$ im Vergleich zur Silbersulfadiazin-Gruppe $(9,27 \pm 1,39)$ signifikant reduziert (LIMA JUNIOR et al., 2019a).

\section{DISKUSSION}

Das Studium der Hauteigenschaften von Tilapia ist ein guter Ausgangspunkt, um sein Potenzial für den Einsatz bei Verbrennungen zu verstehen. Dazu lohnt es sich zunächst, die Ergebnisse von Hu et al. zu analysieren. (2017), der die Verwendung eines Kollagen-Polypeptid-Konzentrats untersuchte, das aus der Haut von Tilapia extrahiert wurde, wobei er sich auf sein heilendes Potenzial konzentrierte. In vitro Assays mit präparierten menschlichen Zellen zeigten, dass die Zugabe von 50,0 $\mu \mathrm{g} / \mathrm{ml}$ des Konzentrats zum verletzten Gewebe statistisch signifikante Ergebnisse in 
Bezug auf die Geschwindigkeit der Regeneration und Reepithelialisierung des Gewebes brachte. Zum anderen zeigten die In vivo Studien desselben Autors vielversprechende Ergebnisse hinsichtlich der Heilung durch tiefe partielle Dickenverbrühung bei den getesteten Kaninchen. Die Ergebnisse ähneln denen, die mit dem Kollagen-Hydrogel-Dressing erzielt wurden, das von $\mathrm{Ge}$ et al. untersucht wurde. (2020), für tiefe Verbrennungen.

Darüber hinaus wurden weitere Studien durchgeführt, um die Eigenschaften der Tierhaut zu bewerten. Nach Alves et al. (2015), Tilapia-Haut präsentiert Bündel dichter Kollagenfasern, überwiegend Typ 1, mit mikroskopischen Eigenschaften, die denen der menschlichen Haut ähneln, sowie hoher Zugfestigkeit und Streckung, was sie als Biomaterial in der regenerativen Medizin vielversprechend macht. Die Studie von Ouyang et al. (2018) bestätigt diese Ergebnisse sowie die von Hu et al. (2017), das sich auch mit der antibakteriellen Aktivität des Materials befasst und seine Aushärtung Effizienz mit anderen auf dem Markt erhältlichen Lösungen verbindet und neben der Migration und Zellproliferation eine hohe Effizienz erzielt.

Validiert dieses heilende Potenzial der Tilapia-Haut, ist es notwendig, die Verwendbarkeit dieses Materials in Form von biologischen Verbänden zu verstehen. Laut der Studie von Lima Junior (2017) haftet Tilapia-Haut bei Testratten erheblich an Brandwunden, mit positiven Ergebnissen während der Heilung, ohne signifikante Mutationen im biochemischen und hämatologischen Bereich der Leber- und Nierenfunktion, was eine positive Möglichkeit als biologischer Verband darstellt. Diese Ergebnisse bestätigend ist die randomisierte klinische Studie von Lima Junior et al. (2020c) zeigte eine gute Adhärenz bei Patienten sowie andere Vorteile gegenüber anderen Verbänden wie aquacel ag ${ }^{\circledR}$, auf die später eingegangen wird.

Das heißt, es lohnt sich, die Tilapia-Haut mit anderen bestehenden Lösungen zu vergleichen, wenn es um Verbrennungen geht. Lima Junior et al. (2020c) und Marcelo und Brandt (2019) förderten Studien, die keine Minderwertigkeit in lyophilisierter Tilapia-Haut zeigen, verglichen mit dem schmutzigen Carboxymethylcellulose-Dressing, das mit Silber imprägniert ist, Aquacel $\mathrm{Ag} \AA$. Die Studie von Lima Junior et al. (2020c) betont, dass die Verwendung von Tilapia-Haut 
eine subjektive Verbesserung der Menge der gemeldeten Schmerzen und der Anzahl der erforderlichen Verbände bietet, wodurch die Behandlungskosten und die Arbeitsbelastung des Teams reduziert werden. Daher ist Tilapia-Haut eine gute Option für die Behandlung von Verbrennungen.

\section{FAZIT}

Angesichts der Ergebnisse der in der vorliegenden Übersicht berichteten Literatur wird der Schluss gezogen, dass Studien mit der Haut von Nil-Tilapia, Süßwasserfischen, die von Forschern der Bundesuniversität Ceará durchgeführt wurden, die seit 2016 die Pioniere waren, um die Methode patentieren zu lassen. Darüber hinaus war es eine revolutionäre Modalität mit zahlreichen Vorteilen bei der Behandlung von Patienten mit oberflächlichen und tiefen Hautläsionen, die sich auf verschiedene Bereiche der Medizin ausdehnen, die in der Veterinärmedizin innerhalb und außerhalb des Landes getestet wurden (LIMA JUNIOR, 2017).

Basierend auf den Forschungsaufzeichnungen werden die Vorteile der Verwendung von Haut-Tilapia bei der Behandlung von Verbrennungen verstärkt, wenn man bedenkt, dass es aufgrund der längeren Periode, die an der Haut des Patienten haftet, eine Verringerung der Schmerzen des Patienten bietet. Neben der reduzierten Infektions- und Kontaminationsgrad, hohe Resistenz aufgrund der großen Menge an Kollagen in der Fischhaut, was zu einer guten Ästhetik und niedrigen Kosten im Vergleich zu anderen Behandlungsformen führt.

Daher kamen die Forscher bei der Beantwortung der Leitfrage zu dem Schluss, dass die neue Methode zwar ein wichtiger Fortschritt bei der Behandlung von Verbrennungen ist, da ihre Beschäftigungsfähigkeit bestätigt wird und sie einen Vorteil gegenüber einigen der wichtigsten bereits bestehenden Alternativen aufweist.

\section{VERWEISE}

ALVES, Ana; VERDE, Maria; FERREIRA FILHO, Antônio; SILVA, Paulo; FEITOSA, Victor; LIMA JUNIOR, Edmar; MIRANDA, Marcelo; MORAES FILHO, Manoel. 
Avaliação microscópica, estudo histoquímico e análise de propriedades tensiométricas da pele de tilápia do Nilo. Revista Brasileira de Queimaduras, v. 14, n. 3, p. 203-210, 2015.

COSTA, Bruno; LIMA JÚNIOR, Edmar; MORAES FILHO, Manoel; FECHINE, Francisco; MORAES, Maria; SILVA JUNIOR, Francisco; SOARES, Maria; ROCHA, Marina. Use of Tilapia Skin as a Xenograft for Pediatric Burn Treatment: A Case Report. Journal of Burn Care \& Research, v. 40, n. 5, p. 714-717, 2019.

GE, Baosheng; WANG, Haonan; LI, Jie; LIU, Hengheng; YIN, Yonghao; ZHANG, Naili; QIN, Song. Comprehensive Assessment of Nile Tilapia Skin (Oreochromis niloticus) Collagen Hydrogels for Wound Dressings. Marine Drugs, v. 18, n. 4, p. $178,2020$.

HU, Zhang; YANG, Ping; ZHOU, Chunxia; LI, Sidong; HONG, Pengzhi. Marine Collagen Peptides from the Skin of Nile Tilapia (Oreochromis niloticus): Characterization and Wound Healing Evaluation. Marine Drugs, v. 15, n. 4, p. 102, 2017.

LEONTSINIS, Cybele; LIMA JUNIOR, Edmar; MORAIS FILHO, Manoel; BRITO, Maria; ROCHA, Marina; NASCIMENTO, Maria; SILVA JUNIOR, Francisco; MIRANDA, Marcelo. Elaboração de um protocolo para implementação e funcionamento do primeiro banco de pele animal do Brasil: Relato de experiência. Revista Brasileira de Queimaduras, v. 17, n. 1, p. 66-71, 2018.

LIMA JUNIOR, Edmar; PICOLLO, Nelson; MIRANDA, Marcelo; RIBEIRO, Wesley; ALVES, Ana; FERREIRA, Guilherme; PARENTE, Ezequiel, MORAES FILHO, Manoel. Uso da pele de tilápia (Oreochromis niloticus), como curativo biológico oclusivo, no tratamento de queimaduras. Revista Brasileira de Queimaduras, v. 16, n. 1 , p. 10-17, 2017. 
LIMA JÚNIOR, Edmar. Tecnologias inovadoras: uso da pele da tilápia do Nilo no tratamento de queimaduras e feridas. Revista Brasileira de Queimaduras, v. 16, n. 1, p. 1-2, 2017.

LIMA JÚNIOR, Edmar; MORAES FILHO, Manoel; FORTE, Antônio; COSTA, Bruno; ; FECHINE, Francisco; ALVES, Ana; MORAES, Maria; ROCHA, Marina; SILVA JUNIOR, Francisco; SOARES, Maria; BEZERRA, Alane; MARTINS, Camila; MATHOR, Mônica. Pediatric Burn Treatment Using Tilapia Skin as a Xenograft for Superficial Partial-Thickness Wounds: A Pilot Study. Journal of Burn Care \& Research, 2019a.

LIMA JUNIOR, Edmar; MORAES FILHO, Manoel; COSTA, Bruno Almeida; FECHINE, Francisco; MORAES, Maria; SILVA JUNIOR, Francisco; SOARES, MARIA; ROCHA, MARINA, LEONTSINIS, Cybele. Innovative treatment using tilapia skin as a xenograft for partial thickness burns after a gunpowder explosion. Journal of Surgical Case Reports, v. 2019, n. 6, $2019 \mathrm{~b}$.

LIMA JÚNIOR, Edmar; MORAES FILHO, Manoel; COSTA, Bruno; ROHLEDER, Andréa; ROCHA, Marina; FECHINE, Francisco; FORTE, Antonio. ALVES, Ana, SILVA JUNIOR, Francisco; MARINS, Camila; MATHOR, Mônica; MORAES, Maria. Innovative Burn Treatment Using Tilapia Skin as a Xenograft: A Phase II Randomized Controlled Trial. Journal of Burn Care \& Research, v. 41, n. 3, p. 585592, 2020a.

LIMA JUNIOR, Edmar; MORAES, Manoel Odorico; COSTA, Bruno Almeida; UCHÔA, Alex; MARTINS, Camila; MORAES, Maria; ROCHA, Marina; FECHINE, Rodrigo. Treatment of deep second-degree burns on the abdomen, thighs, and genitalia: use of tilapia skin as a xenograft. Revista Brasileira de Cirurgia Plástica (RBCP) - Brazilian Journal of Plastic Sugery, v. 35, n. 2, p. 243-248, 2020b.

LIMA JÚNIOR, Edmar; MORAES FILHO, Manoel ; COSTA, Bruno Almeida; FECHINE, Francisco; ROCHA, Marina; VALE, Mariana; DIÓGENES, Ana; 
UCHÔA, Alex; SILVA JUNIOR, Francisco; MARTINS, Camila; BANDEIRA, Tereza; RODRIGUES, Felipe; PAIER, Carlos; MORAES, Maria. A Randomized Comparison Study of Lyophilized Nile Tilapia Skin and Silver-Impregnated Sodium Carboxymethylcellulose for the Treatment of Superficial Partial-Thickness Burns. Journal of Burn Care \& Research, v. 42, n. 1, p. 41-48, 2020 c.

DE MIRANDA, Marcelo José Borges; BRANDT, Carlos Teixeira. Xenoenxerto (pele da Tilápia-do-Nilo) e hidrofibra com prata no tratamento das queimaduras de II grau em adultos. Rev. bras. cir. plást, p. 79-85, 2019.

OUYANG, Qian-Qian; HU, Zhang; LIN, Zhen-Peng; QUAN, Wei-Yan; DENG, YiFeng; LI, Si-Dong; LI, Pu-Wang, CHEN, Yu. Chitosan hydrogel in combination with marine peptides from tilapia for burns healing. International Journal of Biological Macromolecules, v. 112, p. 1191-1198, 2018.

Eingereicht: Juli 2021.

Genehmigt: Dezember 2021. 